\title{
Gender, anthropometric factors and risk of colorectal cancer with particular reference to tumour location and TNM stage: a cohort study
}

\author{
Jenny Brändstedt ${ }^{*}$, Sakarias Wangefjord ${ }^{1}$, Björn Nodin ${ }^{1}$, Alexander Gaber ${ }^{1}$, Jonas Manjer ${ }^{2,3}$ and Karin Jirström ${ }^{1}$
}

\begin{abstract}
Background: It remains unclear whether the increased risk of colorectal cancer (CRC) associated with obesity differs by gender, distribution of fat, tumour location and clinical (TNM) stage. The primary aim of this study was to examine these associations in 584 incident colorectal cancer cases from a Swedish prospective population-based cohort including 28098 men and women.
\end{abstract}

Methods: Seven anthropometric factors; height, weight, bodyfat percentage, hip circumference, waist circumference, BMI and waist-hip ratio (WHR) were categorized into quartiles of baseline anthropometric measurements. Relative risks of CRC, total risk as well as risk of different TNM stages, and risk of tumours located to the colon or rectum, were calculated for all cases, women and men, respectively, using multivariate Cox regression models.

Results: Obesity, as defined by all anthropometric variables, was significantly associated with an overall increased risk of CRC in both women and men. While none of the anthropometric measures was significantly associated with risk of tumour (T)-stage 1 and 2 tumours, all anthropometric variables were significantly associated with an increased risk of T-stage 3 and 4, in particular in men. In men, increasing quartiles of weight, hip, waist, BMI and WHR were significantly associated with an increased risk of lymph node positive (N1 and N2) disease, and risk of both non-metastatic (M0) and metastatic (M1) disease. In women, there were no or weak associations between obesity and risk of node-positive disease, but statistically significant associations between increased weight, bodyfat percentage, hip, BMI and MO disease. Interestingly, there was an increased risk of colon but not rectal cancer in men, and rectal but not colon cancer in women, by increased measures of weight, hip-, waist circumference and bodyfat percentage.

Conclusions: This study is the first to show a relationship between obesity, measured as several different anthropometric factors, and an increased risk of colorectal cancer of more advanced clinical stage, in particular in men. These findings suggest that risk of CRC differs according to the method of characterising obesity, and also according to gender, location, and tumour stage.

\section{Background}

Colorectal cancer (CRC) is the third most common malignancy in developed countries with approximately 1 million new cases every year [1]. Life style factors may contribute to the aetiology of several cancer forms [2], including CRC and, since they are modifiable, a better understanding of the associations between lifestyle-

\footnotetext{
* Correspondence: jenny.brandstedt@med.lu.se

${ }^{1}$ Department of Clinical Sciences, Division of Pathology, Lund University,

Skåne University Hospital, Lund SE-221 85, Sweden

Full list of author information is available at the end of the article
}

related factors and CRC risk may be of importance in order to develop novel strategies for prevention of the disease.

Numerous epidemiological studies and meta-analyses have examined the relationship between body weight and body mass index (BMI) and CRC, mainly risk of colon cancer, and most studies have shown a positive relationship between a high BMI and risk of colon cancer in men, whereas weak or no associations were reported in women [3-16]. The reasons for the apparently discrepant associations between BMI and CRC risk in men

\section{Biomed Central}


and women remain unclear. Due to inconsistencies in the definition of obesity, the results of epidemiologic studies may be somewhat difficult to generalize, although the majority of studies have used BMI as the principal measure of adiposity. The discrepancy between men and women in the association of body weight with CRC may be related to differences in fat distribution between the sexes. A high BMI in men is more closely related to central/abdominal obesity, whereas in women, a high BMI more often correlates with lower body obesity, measured as hip circumference [17]. Some prospective studies have investigated the association of body fat distribution measured as waist-, hip circumference and waist-hip ratio (WHR) and CRC risk [6,8,12,13,18-23]. All of these studies found waist circumference to be associated with an increased risk. However, in most of these studies, waist- and hip measurements were selfreported rather than measured $[8,20,23]$. One large study [6] has shown that both waist circumference and WHR are strongly related to CRC risk in both men and women, supporting the hypothesis that abdominal obesity is a risk factor for CRC in both sexes and suggesting that fat distribution is more important than body weight or BMI in women.

A small number of studies have examined associations of other anthropometric factors than BMI and CRC risk according to tumour location; i.e. colon or rectum $[4,6,14,22]$. Most of these studies found no association with body weight or BMI according to location, while one study has shown an increased risk of rectal cancer in overweight/obese men [14].

To our knowledge, only two previous studies $[13,18]$ have addressed the question of whether obesity is associated with an increased risk of developing CRC of different clinical (TNM) stages, based on the extent of the tumour $(\mathrm{T})$, the extent of spread to the lymph nodes $(\mathrm{N})$, and the presence of distant metastasis $(\mathrm{M})$. The aim of this study was therefore to examine these associations in participants in the Malmö Diet and Cancer Study (MDCS), a large prospective population based Swedish cohort study. A further aim was to examine whether these associations differ between men and women.

\section{Methods}

\section{The Malmö Diet and Cancer Study}

The Malmö Diet and Cancer Study (MDCS) is a population-based prospective cohort study, forming part of the European Prospective Investigation into Cancer and $\mathrm{Nu}$ trition (EPIC)[24] that enrolled 28098 individuals during baseline examinations between 1991 and 1996 [25].

The main purpose of the MDCS was to further investigate the associations between a Western diet rich in fat and low in fruit and vegetables and certain forms of cancer. Participants in the MDCS and EPIC cohort include
11063 men (39.4\%) and 17035 women (60.6\%) between 44 and 74 years of age. Data on lifestyle, health and socio-demographic characteristics were collected via standardized self-administered questionnaires. Ethical permission for the MDCS (LU 90-51) and the present study (LU 530-2008) was obtained from the Ethics Committee at Lund University.

\section{Anthropometric measurements}

At baseline examination, weight (multiples of $0.1 \mathrm{~kg}$ ) and height (to the nearest $0.005 \mathrm{~m}$ ) was measured by a trained nurse, and body mass index (BMI) was calculated as $\mathrm{kg} / \mathrm{m}^{2}$. Waist circumference was measured at the midpoint between the lower ribs and the iliac crest, and for hip circumference the level of greatest lateral extension was used. These measurements were estimated to the nearest $0.01 \mathrm{~m}$. The waist and hip circumferences of each participant were used to calculate waist-hip ratio (WHR; $\mathrm{cm} / \mathrm{cm}$ ) as an additional measure of fat distribution. Body composition was estimated using a single frequency bio-impedance methodology (BIA 103, RLJ-systems, Detroit, MI, USA) with tetra polar electrode placement and subjects in a supine position. Lean body mass and fat mass were determined and served to calculate body fat percentage. The BIA method has previously been validated in Swedish middle-aged and elderly adults [26].

\section{Follow-up for cancer and cause-of-death}

Incident cases of invasive colorectal cancer in the MDCS were identified through the Swedish Cancer Registry and vital status was determined by record linkage with the Swedish Cause-of -Death Registry. End of follow-up was 31 December 2009. Information on vital status and cause of death was obtained from the Swedish Cause of Death Registry until 31 December 2009. Time on study was defined as time from baseline to diagnosis, death or end of follow-up 31 December 2009. Median time from baseline until diagnosis was $8.6(\mathrm{SD}=4.3)$ years and the median follow-up time in the entire cohort was 13.7 $(\mathrm{SD}=3.2)$ years.

\section{Study population}

Among the 28098 men and women in the cohort, there was a total number of 584 cases of incident colorectal cancer. Eight tumours were re-classified as tumour in situ (TIS), and these were not included as cases. A total number of 181 cases were diagnosed with CRC before baseline examination, i.e prevalent colorectal cancers, and therefore excluded from the study. Cases with other prevalent cancers were not excluded from the study.

\section{Tumour characteristics}

Patient and tumour characteristics in the cohort have been described in detail previously [27-30]. The distribution of 
clinicopathological characteristics did not differ between all CRC cases in the MDCS $(n=626)$ and cases included in the EPIC cohort $(n=584)$ (data not shown). Of all incident CRC cases, 337 (59\%) had tumours located in the colon and $216(38 \%)$ had tumours located in the rectum. Cases with multiple synchronous location $(\mathrm{n}=14)$, and cases with unknown location $(n=17)$ were excluded from the analysis related to tumour location. All tumours were histopathologically re-evaluated by a senior pathologist (KJ). According to the TNM classification, 51 (9.7\%) cases presented in T-stage 1, 62 (11.8\%) in T-stage 2, 323 (61.3\%) in T-stage 3 and $82(15.6 \%)$ in T-stage 4 . Two hundred and ninety two (58.4\%) cases presented with lymph node negative (N0) disease, 124 (24.8\%) had N1 (1-3 positive lymph nodes) and 84 (16.8\%) N2 (4 or more positive lymph nodes) disease. Four hundred and fifty (79.5\%) patients did not have distant metastases (M0), and 116 (20.4\%) had M1 disease. The distribution of clinicopathological characteristics and prognostic factors as well as adjuvant and palliative treatment did not differ between men and women in the cohort [27].

\section{Statistical methods}

Distribution of established and potential risk factors for CRC was compared between CRC cases and the rest of the study cohort. Anthropometric measurements were divided into quartiles. Separate quartiles were calculated for men and women for this analysis (Additional file 1). A Cox proportional hazards analysis was used in order to compare risk of colorectal cancer between different categories of anthropometric factors in both sexes, and for women and men separately. This yielded relative risks (RR) with a 95\% confidence interval. Time on study was used as the underlying time scale, defined as time from baseline to diagnosis, death or end of follow-up 31 December 2009. The proportional hazards assumption was confirmed by a log, - log plot [31]. In the multivariate Cox analysis potential confounders were included, i.e age (years), educational level (not completed school/ elementary school (6-8 years)/ "grundskola" (9-10 years)/ "studentexamen" (10-12 years)/ one year after "studentexamen"/ university degree), smoking habits (yes regularly, yes occasionally, former smoker, never smoker), and alcohol consumption (g/day) (Table 1). All statistical analyses were conducted using SPSS version 16 (SPSS Inc., Chicago, IL, USA). A two-tailed p-value less than 0.05 was regarded as statistically significant.

\section{Results}

Baseline characteristics

The distribution of covariates and mean values for the anthropometric measures for men and women who developed CRC during follow-up (cases), and for those who did not (rest of cohort) is presented in Table 1. Cases were slightly older than the rest of cohort, and men were generally older than women in the rest of cohort, but among cases men were slightly younger than women. Mean value for all anthropometric factors were higher in cases as compared to the rest of cohort. Among cases, $47.9 \%$ were men, and $52.1 \%$ women.

\section{Overall risk of colorectal cancer in relation to anthropometric factors}

As shown in Table 2, there was a statistically significant association between all anthropometric factors except bodyfat percentage and an increased risk of CRC in both sexes. In men, strong, statistically significant associations were seen for all factors except height. In women, BMI, hip and weight, but not the other anthropometric measures, were associated with an increased risk of CRC.

\section{Relative risk of colorectal cancer in relation to $\mathrm{T}, \mathrm{N}$ and M-stage}

As shown in Table 3, there were no associations between any of the anthropometric measures and T-stage 1 and 2 . However, weight, hip, waist, BMI and WHR were strongly associated with an increased risk of tumours with T-stage 3 and 4 in both sexes (Table 3). The associations between obesity and more advanced tumour stage (T-stage 3 and 4) were more evident in men (Tables 4 and 5), with a significant relationship between all anthropometric factors, except height, and more advanced T-stage in men, while this relationship was only significant for weight, bodyfat percentage and BMI in women (Tables 4 and 5). Regarding N-stage, there was an overall increased risk of $\mathrm{N} 1$ and N2-tumours for all anthropometric factors except bodyfat percentage and height (Table 3). Subgroup analysis according to gender revealed that these associations were only statistically significant in men (Table 4) and not, except for bodyfat percentage, in women (Table 5). The overall associations between obesity and M-stage were stronger for M0 compared to M1 disease (Table 3). Similar findings were seen among women, where no anthropometric factors were associated with M1 disease (Table 4), while in men, weight, waist and WHR were statistically significant associated with both M0 and M1 disease (Table 4).

\section{Relative risk of colon versus rectal cancer}

Separate analysis of risk of colon and rectal cancer demonstrated an overall increased risk of colon cancer for all anthropometric factors except for bodyfat percentage (Tables 6 and 7). Weight, hip, waist and BMI were significantly associated with an increased risk of rectal cancer. Among men, there was an increased risk of colon but not rectal cancer. In women, no association could be found between anthropometric factors and colon cancer risk, but a statistically significant association was seen 
Table 1 Distribution of risk factors in cases and rest of cohort

\begin{tabular}{|c|c|c|c|c|c|c|c|}
\hline $\begin{array}{l}\text { Factor (number of subjects } \\
\text { with information) }\end{array}$ & Category & $\begin{array}{c}\text { CRC cases } \\
(n=584)\end{array}$ & $\begin{array}{c}\text { Cases, men } \\
(\mathrm{n}=280)\end{array}$ & $\begin{array}{c}\text { Cases, women } \\
(\mathrm{n}=304)\end{array}$ & $\begin{array}{l}\text { Rest of cohort } \\
(\mathrm{n}=\mathbf{2 7 5 1 4})\end{array}$ & $\begin{array}{c}\text { Men } \\
(n=10783)\end{array}$ & $\begin{array}{c}\text { Women } \\
(n=16731)\end{array}$ \\
\hline \multicolumn{8}{|c|}{ Percent* (mean and SD in italics) $^{*}$} \\
\hline Age at baseline & years & $61.8(6.8)$ & $61.7(6.7)$ & $62.1(6.8)$ & $58.0(7.6)$ & $59.2(7.1)$ & $57.2(7.9)$ \\
\hline \multirow[t]{6}{*}{ Education (28027) } & Not completed school & 0.9 & 0.7 & 0.9 & 0.8 & 0.8 & 0.8 \\
\hline & $6-8$ years & 49.1 & 48.5 & 46.8 & 41.0 & 45.0 & 36.1 \\
\hline & $9-10$ years & 22.4 & 19.6 & 22.8 & 26.2 & 19.6 & 28.6 \\
\hline & $10-12$ years & 8.7 & 10.4 & 6.8 & 8.9 & 11.9 & 6.6 \\
\hline & 1 year university & 7.7 & 6.8 & 8.3 & 8.7 & 9.3 & 7.9 \\
\hline & University degree & 10.8 & 13.6 & 7.7 & 14.3 & 13.2 & 14.2 \\
\hline \multirow[t]{4}{*}{ Smoking status (28086) } & Regularly & 21.2 & 18.9 & 23.4 & 23.8 & 23.9 & 22.5 \\
\hline & Occasionally & 3.4 & 4.3 & 2.6 & 4.5 & 4.8 & 4.1 \\
\hline & Former smoker & 39.7 & 51.8 & 28.6 & 33.7 & 43.0 & 26.2 \\
\hline & Never smoked & 35.6 & 25.0 & 45.4 & 37.9 & 28.3 & 41.7 \\
\hline $\begin{array}{l}\text { Alcohol consumption } \\
\text { (28098) }\end{array}$ & g/day & $10.8(14.2)$ & $15.7 / 17.4)$ & $6.2(8.1)$ & $10.7(12.7)$ & $15.5(16.0)$ & $7.7(8.7)$ \\
\hline Heigh (28057)t & $\mathrm{cm}$ & 169.5(8.9) & 176.3(6.4) & $163.3(5.5)$ & 168.6(8.8) & $176.4(6.6)$ & $163.6(6.1)$ \\
\hline Weight (28056) & $\mathrm{kg}$ & $76.6(14.2)$ & $83.8(12.6)$ & $70.0(12.2)$ & $73.4(13.6$ & $81.7(12.1)$ & $68.0(11.7)$ \\
\hline $\begin{array}{l}\text { Bodyfat percentage } \\
\text { (27925) }\end{array}$ & $\%$ & $26.9(7.1)$ & $21.5(5.0)$ & $31.9(4.8)$ & $26.8(7.0)$ & $20.7(5.0)$ & $30.8(5.0)$ \\
\hline Hip (28044) & $\mathrm{cm}$ & 100.7(9.0) & 101.2(7.4) & $100.2(10.2)$ & $98.4(8.8)$ & $99.3(7.1)$ & $97.8(9.9)$ \\
\hline Waist (28045) & $\mathrm{cm}$ & $87.9(13.7)$ & $96.3(10.7)$ & $80.1(11.3)$ & $84.1(14.5)$ & $93.7(12.5)$ & $77.9(12.1$ \\
\hline Bodymass index (28056) & $\mathrm{kg} / \mathrm{m}^{2}$ & $26.6(4.0)$ & $26.9(3.6)$ & $26.3(4.4)$ & $25.7(4.0)$ & $26.2(3.5)$ & $25.4(4.2)$ \\
\hline Waist-hip ratio (28043) & $\mathrm{cm} / \mathrm{cm}$ & $0.87(0.09)$ & 0.9510 .06 & $0.80(0.05)$ & $0.85(0.14)$ & $0.94(0.10$ & $0.80(0.14)$ \\
\hline
\end{tabular}

between weight, bodyfat, hip, and waist and risk of rectal cancer (Tables 6 and 7).

Among women, all anthropometric factors except height and WHR were significantly associated with risk of a more advanced T-stage in rectal but not colon cancer, while there were no associations with risk of more advanced $\mathrm{N}$-stage, neither in colon nor rectal cancer (data not shown). In addition, there was a significant association between height and M1 disease in colon but not rectal cancer in women (data not shown). In men, there were no associations between anthropometric factors and risk of more advanced T-stage in rectal cancer, but in colon cancer, only height was associated with a statistically significant increased risk of T-stage 3 \& 4 disease (data not shown). Moreover, among men, there was a statistically significant increased risk of developing N1-2 tumours related to height, weight, waist and hip in colon and to hip, BMI and WHR in rectal cancer, and a statistically significant association between BMI and M1 disease in colon but not rectum (data not shown).

\section{Discussion}

The results from this large prospective cohort study indicate that the association between obesity and risk of CRC varies by gender, cancer site and tumour aggressiveness. All anthropometric factors except bodyfat percentage were significantly associated with an increased risk of CRC in general, which corresponds well to previous findings $[3,4,6,12,13,15,18,19,32]$. When studying men and women separately, we found an increased risk of CRC for all anthropometric variables, except for height, in men, which is also consistent with previous results [4-6,12,15,16]. However, the finding of a stronger association of obesity and risk of being diagnosed with clinically more advanced CRC in men has, to our knowledge, not been demonstrated before. We also found an overall increased risk of CRC with obesity defined as weight, BMI and WHR in women, which has only been shown in a few previous studies $[4,10,15,18,20]$.

To our knowledge, only two previous studies $[13,18]$ have examined the associations between anthropometric factors and risk of CRC according to tumour aggressiveness, as reflected in TNM-classification of the disease. These two studies examined the association between anthropometry and risk of colon cancer according to late (Stage III and IV) vs early (Stage I and II) stage colon cancer in men and women, respectively, and neither found any statistically significant associations.

We have thus been able to demonstrate an increased risk of more advanced tumours with increased anthropometric measures, particularly in men, among whom several anthropometric variables were significantly associated with risk of a more advanced T-stage, N2 disease, 
Table 2 Anthropometric factors and relative risk of colorectal cancer in all cases, women and men

\begin{tabular}{|c|c|c|c|c|c|c|}
\hline $\begin{array}{l}\text { Anthropometric } \\
\text { factor (quartiles) }\end{array}$ & (n) & $\begin{array}{c}\text { RR all } \\
n=584\end{array}$ & (n) & $\begin{array}{l}\text { RR men } \\
n=280\end{array}$ & (n) & $\begin{array}{c}\text { RR women } \\
n=304\end{array}$ \\
\hline \multirow[t]{4}{*}{ Height } & 127 & 1.00 & 60 & 1.00 & 58 & 1.00 \\
\hline & 130 & $0.99(0.77-1.27)$ & 70 & $1.28(0.91-1.82)$ & 99 & $1.24(0.89-1.71)$ \\
\hline & 154 & $1.17(0.92-1.49)$ & 75 & 1.08(0.77-1.52) & 77 & $1.33(0.95-1.88)$ \\
\hline & 173 & $1.28(1.00-1.63)$ & 75 & $1.28(0.90-1.82)$ & 68 & $1.29(0.89-1.85)$ \\
\hline$p$-trend & & 0.021 & & 0.327 & & 0.160 \\
\hline \multirow[t]{4}{*}{ Weight } & 108 & 1.00 & 52 & 1.00 & 45 & 1.00 \\
\hline & 130 & $1.23(0.95-1.59)$ & 67 & $1.22(0.85-1.76)$ & 83 & $1.58(1.10-2.27)$ \\
\hline & 150 & $1.21(0.94-1.56)$ & 67 & $1.25(0.87-1.80)$ & 83 & $1.83(1.27-2.65)$ \\
\hline & 196 & $1.61(1.26-2.05)$ & 94 & $1.78(1.26-2.52)$ & 92 & $1.68(1.17-2.41)$ \\
\hline$p$-trend & & $<0.001$ & & 0.001 & & 0.007 \\
\hline \multirow[t]{4}{*}{ Bodyfatpercentage } & 131 & 1.00 & 41 & 1.00 & 51 & 1.00 \\
\hline & 145 & $1.07(0.84-1.36)$ & 58 & $1.10(0.74-1.64)$ & 58 & $1.09(0.74-1.59)$ \\
\hline & 122 & $0.90(0.70-1.16)$ & 91 & $1.25(0.87-1.82)$ & 81 & $1.33(0.93-1.90)$ \\
\hline & 186 & $1.06(0.84-1.34)$ & 88 & $1.54(1.06-2.23)$ & 113 & $1.30(0.92-1.82)$ \\
\hline$p$-trend & & 0.858 & & 0.012 & & 0.086 \\
\hline \multirow[t]{4}{*}{ Hip } & 96 & 1.00 & 47 & 1.00 & 45 & 1.00 \\
\hline & 118 & 1.03(0.79-1.36) & 54 & 1.08(0.73-1.60) & 70 & $1.11(0.76-1.62)$ \\
\hline & 170 & $1.43(1.11-1.84)$ & 86 & $1.65(1.15-2.36)$ & 82 & $1.27(0.88-1.84)$ \\
\hline & 200 & $1.41(1.10-1.81)$ & 93 & $1.48(1.03-2.11)$ & 106 & $1.39(0.97-1.99)$ \\
\hline P-trend & & 0.001 & & 0.007 & & 0.042 \\
\hline \multirow[t]{4}{*}{ Waist } & 94 & 1.00 & 52 & 1.00 & 44 & 1.00 \\
\hline & 140 & $1.31(1.01-1.71)$ & 44 & $0.75(0.50-1.12)$ & 71 & $1.15(0.79-1.69)$ \\
\hline & 125 & 1.03(0.78-1.35) & 84 & $1.32(0.93-1.88)$ & 99 & $1.46(1.02-2.08)$ \\
\hline & 225 & $1.76(1.37-2.27)$ & 100 & $1.65(1.17-2.31)$ & 89 & $1.29(0.89-1.86)$ \\
\hline$P$ - trend & & $<0.001$ & & $<0.001$ & & 0.114 \\
\hline \multirow[t]{4}{*}{ BMl } & 99 & 1.00 & 64 & 1.00 & 48 & 1.00 \\
\hline & 124 & $1.25(0.96-1.63)$ & 49 & $0.73(0.50-1.06)$ & 82 & $1.53(1.07-2.19)$ \\
\hline & 162 & $1.22(0.94-1.57)$ & 67 & $0.94(0.67-1.33)$ & 81 & $1.43(1.00-2.05)$ \\
\hline & 199 & $1.69(1.32-2.17)$ & 100 & $1.61(1.17-2.22)$ & 92 & $1.61(1.12-2.30)$ \\
\hline P-trend & & $<0.001$ & & 0.001 & & 0.027 \\
\hline \multirow[t]{4}{*}{ WHR } & 124 & 1.00 & 66 & 1.00 & 77 & 1.00 \\
\hline & 127 & $1.02(0.79-1.30)$ & 47 & $0.99(0.68-1.44)$ & 65 & $1.02(0.73-1.42)$ \\
\hline & 145 & $1.06(0.83-1.36)$ & 81 & 1.36(0.98-1.88) & 81 & $1.04(0.76-1.43)$ \\
\hline & 187 & $1.49(1.17-1.89)$ & 86 & $1.64(1.19-2.28)$ & 80 & $1.14(0.83-1.57)$ \\
\hline P-trend & & 0.001 & & 0.001 & & 0.413 \\
\hline
\end{tabular}

Adjusted for age at baseline, level of education, smoking habits, alcohol consumption.

and both M0 and M1-tumours. In women, several anthropometric variables were associated with an increased risk of more advanced T-stage, but not node-positive disease. Furthermore, increased anthropometric measures were only associated with non-metastatic (M0) disease in women. Taken together, these findings suggest that overweight persons have an increased risk of developing more advanced colorectal cancer forms, but that this risk may differ according to gender.
Moreover, our findings provide further evidence of gender-related differences in tumour susceptibility of cancer in the colon and rectum, respectively, as we found a positive association between obesity and risk of developing colon cancer in men and rectal cancer in women. This has to our knowledge not been shown previously, since only a few studies have examined risk of colon and rectal cancer separately. However, two previous studies $[4,14]$ have shown a positive relationship 
Table 3 Anthropometric factors and relative risk of colorectal cancer of different $\mathrm{T}$, $\mathrm{N}$ - and $\mathbf{M}$ stages in all cases

\begin{tabular}{|c|c|c|c|c|c|c|c|c|c|c|c|c|}
\hline $\begin{array}{l}\text { Anthropometric } \\
\text { factor (quartiles) }\end{array}$ & (n) & $\begin{array}{l}\text { RR of } \\
\text { T-stage } 1 \& 2 \\
n=113\end{array}$ & (n) & $\begin{array}{l}\text { RR of } \\
\text { T-stage } 3 \& 4 \\
n=405\end{array}$ & (n) & $\begin{array}{l}\text { RR of } \\
\mathrm{N} \text {-stage } 0 \\
\mathrm{n}=292\end{array}$ & (n) & $\begin{array}{l}\text { RR of } \\
N \text {-stage } 1 \& 2 \\
n=208\end{array}$ & (n) & $\begin{array}{l}\text { RR of } \\
M \text {-stage } 0 \\
n=450\end{array}$ & (n) & $\begin{array}{l}\text { RR of } \\
M \text {-stage } 1 \\
n=116\end{array}$ \\
\hline \multirow[t]{4}{*}{ Height } & 25 & 1.00 & 91 & 1.00 & 61 & 1.00 & 46 & 1.00 & 92 & 1.00 & 35 & 1.00 \\
\hline & 26 & $0.97(0.56-1.68)$ & 92 & $1.00(0.74-1.33)$ & 68 & $1.10(0.78-1.57)$ & 49 & $0.99(0.66-1.49)$ & 109 & $1.12(0.83-1.47)$ & 18 & $0.51(0.29-0.91)$ \\
\hline & 33 & $1.00(0.58-1.74)$ & 102 & $1.12(0.83-1.49)$ & 83 & $1.33(0.95-1.87)$ & 38 & $0.80(0.51-1.23)$ & 118 & $1.12(0.84-1.49)$ & 26 & $0.76(0.45-1.28)$ \\
\hline & 29 & 1.06(0.61-1.85) & 120 & $1.29(0.96-1.72)$ & 80 & $1.24(0.87-1.77)$ & 75 & $1.52(1.03-2.24)$ & 132 & 1.32(0.98-1.78) & 37 & $1.02(0.62-1.68)$ \\
\hline$p$-trend & & 0.807 & & 0.060 & & 0.153 & & 0.055 & & 0.037 & & 0.678 \\
\hline \multirow[t]{4}{*}{ Weight } & 17 & 1.00 & 77 & 1.00 & 60 & 1.00 & 37 & 1.00 & 80 & 1.00 & 26 & 1.00 \\
\hline & 38 & $2.21(1.24-3.93)$ & 78 & $1.03(0.75-1.42)$ & 66 & $1.11(0.78-1.58)$ & 40 & $1.12(0.72-1.76)$ & 111 & $1.09(0.84-1.41)$ & 16 & $0.64(0.35-1.20)$ \\
\hline & 26 & $1.26(0.68-2.34)$ & 109 & $1.24(0.92-1.66)$ & 71 & $1.00(0.71-1.42)$ & 55 & 1.34(0.88-2.03) & 109 & $1.23(0.94-1.61)$ & 36 & $1.22(0.73-2.04)$ \\
\hline & 32 & $1.52(0.83-2.78)$ & 141 & $1.64(1.23-2.18)$ & 95 & 1.35(0.97-1.89) & 76 & $1.87(1.25-2.80)$ & 151 & $1.61(1.25-2.09)$ & 38 & $1.31(0.78-2.20)$ \\
\hline$p$-trend & & 0.673 & & $<0.001$ & & 0.116 & & 0.001 & & 0.002 & & 0.100 \\
\hline \multirow[t]{4}{*}{ Body fat percentage } & 25 & 1.00 & 86 & 1.00 & 71 & 1.00 & 37 & 1.00 & 96 & 1.00 & 31 & 1.00 \\
\hline & 27 & $1.10(0.61-1.82)$ & 104 & $1.17(088-1.56)$ & 67 & $0.92(0.66-1.29)$ & 61 & $1.56(1.04-2.35)$ & 115 & 1.13(0.72-1.79) & 28 & $0.88(0.53-1.46)$ \\
\hline & 25 & $1.04(0.59-1.84)$ & 82 & $0.92(0.67-1.25)$ & 61 & $0.86(0.61-1.22)$ & 42 & $1.07(0.68-1.67)$ & & 1.24(0.82-1.88) & 24 & $0.75(0.44-1.30)$ \\
\hline & 36 & $1.21(0.71-2.06)$ & 133 & $1.13(0.85-1.50)$ & 93 & $0.99(0.72-1.37)$ & 68 & $1.36(0.90-2.05)$ & 91 & $1.17(0.80-1.70)$ & & $0.76(0.45-1.27)$ \\
\hline$p$-trend & & 0.498 & & 0.706 & & 0.948 & & 0.460 & 149 & 0.483 & 33 & 0.261 \\
\hline \multirow[t]{4}{*}{ Hip } & 19 & 1.00 & 65 & 1.00 & 56 & 1.00 & 30 & 1.00 & 74 & 1.00 & 22 & 1.00 \\
\hline & 21 & $0.92(0.49-1.71)$ & 53 & $1.02(0.74-1.42)$ & 53 & $0.77(0.54-1.15)$ & 41 & $1.18(0.74-1.90)$ & 93 & $1.08(0.80-1.44)$ & 18 & $0.68(0.37-1.27)$ \\
\hline & 32 & $1.30(0.73-2.32)$ & 119 & $1.48(1.09-2.01)$ & 84 & 1.18(0.84-1.67) & 61 & $1.74(1.12-2.71)$ & 122 & $1.47(1.11-1.93)$ & 41 & $1.52(0.90-2.56)$ \\
\hline & 41 & $1.46(0.84-2.55)$ & 142 & $1.46(1.08-1.98)$ & 99 & $1.17(0.84-1.63)$ & & $1.80(1.17-2.77)$ & 162 & 1.52(1.18-1.95) & 35 & $1.04(0.60-1.79)$ \\
\hline$p$-trend & & 0.077 & & 0.002 & & 0.089 & & 0.002 & & 0.001 & & 0.323 \\
\hline \multirow[t]{4}{*}{ Waist } & 19 & 1.00 & 62 & 1.00 & 49 & 1.00 & 33 & 1.00 & 70 & 1.00 & 21 & 1.00 \\
\hline & & $1.45(0.81-2.60)$ & 91 & $1.28(0.93-1.78)$ & 71 & $1.23(0.85-1.78)$ & 45 & $1.23(0.79-1.94)$ & 109 & $0.81(0.60-1.09)$ & 23 & $0.95(0.52-1.71)$ \\
\hline & 31 & $0.98(0.53-1.81)$ & 85 & $1.06(0.76-1.48)$ & 57 & $0.85(0.58-1.26)$ & 44 & $1.09(0.69-1.73)$ & 97 & $1.38(1.07-1.79)$ & 25 & $0.90(0.50-1.63)$ \\
\hline & 25 & $1.36(0.75-2.43)$ & 167 & $2.00(1.49-2.72)$ & 115 & $1.60(1.13-2.27)$ & 86 & $2.10(1.38-3.18)$ & 175 & $1.70(1.32-2.19)$ & 47 & $1.63(0.96-2.80)$ \\
\hline$p$-trend & 38 & 0.596 & & $<0.001$ & & 0.022 & & 0.001 & & $<0.001$ & & 0.049 \\
\hline \multirow[t]{4}{*}{ BMI } & 22 & 1.00 & 64 & 1.00 & 51 & 1.00 & 34 & 1.00 & 74 & 1.00 & 23 & 1.00 \\
\hline & 28 & $1.22(0.69-2.15)$ & 78 & $1.22(0.87-1.70)$ & 69 & $1.33(0.92-1.92)$ & 39 & $1.18(0.75-1.88)$ & 99 & $1.19(0.91-1.56)$ & 21 & $0.93(0.51-1.68)$ \\
\hline & 32 & $1.05(0.60-1.81)$ & 111 & $1.29(0.94-1.75)$ & 73 & $1.03(0.72-1.48)$ & 59 & 1.34(0.87-2.65) & 127 & $1.10(0.83-1.45)$ & 29 & $0.95(0.55-1.65)$ \\
\hline & 31 & $1.16(0.66-2.03)$ & 152 & $1.97(1.46-2.66)$ & 99 & $1.56(1.10-2.20)$ & 76 & 1.98(1.31-2.99) & 151 & $1.63(1.27-2.10)$ & 43 & $1.59(0.95-2.67)$ \\
\hline$p$-trend & & 0.773 & & $<0.001$ & & 0.043 & & 0.001 & & $<0.001$ & & 0.063 \\
\hline \multirow[t]{4}{*}{ WHR } & 28 & 1.00 & 81 & 1.00 & 65 & 1.00 & 43 & 1.00 & 101 & 1.00 & 20 & 1.00 \\
\hline & 25 & $0.90(0.52-1.56)$ & 87 & $1.05(0.78-1.43)$ & 58 & $0.85(0.60-1.22)$ & 48 & 1.14(0.75-1.72) & 91 & $1.01(0.73-1.41)$ & 29 & $1.40(0.79-2.48)$ \\
\hline & 30 & $0.91(0.54-1.55)$ & 97 & $1.10(0.81-1.48)$ & 83 & $1.10(0.79-1.53)$ & 39 & $0.88(0.56-1.36)$ & 117 & $1.23(0.92-1.64)$ & 26 & $1.20(0.66-2.16)$ \\
\hline & 30 & $0.89(0.51-1.56)$ & 139 & $1.74(1.30-2.33)$ & 86 & $1.19(0.84-1.67)$ & 77 & $1.95(1.32-2.89)$ & 141 & 1.58(1.19-2.09) & 41 & $2.10(1.20-3.69)$ \\
\hline$p$-trend & & 0.707 & & $<0.001$ & & 0.176 & & 0.003 & & 0.013 & & 0.017 \\
\hline
\end{tabular}

Adjusted for age, level of education, smoking habits and alcohol consumption.

between BMI, overweight and rectal cancer risk in men, which is in contrast to our findings. Moreover, subgroup analysis related to risk of more advanced disease in colon and rectal cancer, respectively, also revealed a more pronounced association between anthropometric factors and risk of advanced disease in men.

Body weight or BMI have been the most commonly used anthropometric measurements to examine the associations of obesity and colorectal cancer risk in previous studies, the majority of which have shown a positive relationship between BMI and risk of CRC in men, but week or no associations in women [3-16]. However, these measurements may not be ideal because of the changes in physiologic functions that to a certain extent depend on regional adipose tissue distribution [33]. Some prospective studies have examined the association of body fat 
Table 4 Anthropometric factors and relative risk of colorectal cancer of different $T$, N and $M$ stages in men

\begin{tabular}{|c|c|c|c|c|c|c|c|c|c|c|c|c|}
\hline $\begin{array}{l}\text { Anthropometric } \\
\text { factor (quartiles) }\end{array}$ & (n) & $\begin{array}{l}\text { RR of } \\
\text { T-stage } 1 \& 2 \\
n=50\end{array}$ & (n) & $\begin{array}{l}\text { RR of } \\
\text { T-stage } 3 \& 4 \\
n=199\end{array}$ & (n) & $\begin{array}{l}\text { RR of } \\
N \text {-stage } 0 \\
n=140\end{array}$ & (n) & $\begin{array}{l}\text { RR of } \\
N \text {-stage } 1 \& 2 \\
n=102\end{array}$ & (n) & $\begin{array}{l}\text { RR of } \\
M \text {-stage } 0 \\
n=214\end{array}$ & (n) & $\begin{array}{l}\text { RR of } \\
\text { M-stage } 1 \\
n=60\end{array}$ \\
\hline \multirow[t]{4}{*}{ Height } & 9 & 1.00 & 48 & 1.00 & 37 & 1.00 & 16 & 1.00 & 49 & 1.00 & 11 & 1.00 \\
\hline & 11 & $1.24(0.51-3.01)$ & 47 & $1.09(0.73-1.63)$ & 34 & $1.02(0.64-1.62)$ & 21 & $1.42(0.74-2.72)$ & 51 & $1.14(0.77-1.69)$ & 17 & $1.75(0.82-3.74)$ \\
\hline & 18 & $1.56(0.69-3.49)$ & 47 & $0.87(0.58-1.31)$ & 31 & $0.74(0.46-1.21)$ & 33 & $1.72(0.94-3.14)$ & 58 & $1.02(0.69-1.50)$ & 13 & $1.07(0.48-2.40)$ \\
\hline & 12 & $1.13(0.47-2.75)$ & 57 & $1.29(0.87-1.91)$ & 38 & $1.10(0.69-1.77)$ & 32 & $1.93(1.04-3.56)$ & 56 & $1.16(0.78-1.73)$ & 19 & $1.94(0.90-4.16)$ \\
\hline$p$-trend & & 0.677 & & 0.406 & & 0.978 & & 0.029 & & 0.600 & & 0.226 \\
\hline \multirow[t]{4}{*}{ Weight } & 13 & 1.00 & 33 & 1.00 & 32 & 1.00 & 11 & 1.00 & 43 & 1.00 & 9 & 1.00 \\
\hline & 11 & $0.77(0.34-1.72)$ & 51 & $1.48(0.95-2.29)$ & 34 & $1.00(0.62-1.62)$ & 26 & $2.23(1.10-4.53)$ & 48 & $1.05(0.69-1.58)$ & 18 & $2.02(0.90-4.51)$ \\
\hline & 12 & $0.82(0.37-1.82)$ & 47 & $1.41(0.90-2.20)$ & 35 & $1.06(0.65-1.72)$ & 24 & $2.06(1.01-4.22)$ & 56 & $1.25(0.83-1.86)$ & 11 & $1-31(0.54-3.17)$ \\
\hline & 14 & $0.90(0.42-1.96)$ & 68 & $2.09(1.37-3.19)$ & 39 & $1.19(0.74-1.91)$ & 41 & $3.58(1.82-7.01)$ & 67 & $1.50(1.02-2.22)$ & 22 & $2.75(1.25-6.04)$ \\
\hline$p$-trend & & 0.867 & & 0.001 & & 0.448 & & $<0.001$ & & 0.023 & & 0.032 \\
\hline \multirow[t]{5}{*}{ Body fat percentage } & 8 & 1.00 & 29 & 1.00 & 24 & 1.00 & 10 & 1.00 & 30 & 1.00 & 10 & 1.00 \\
\hline & 9 & $0.89(0.34-2.32)$ & 40 & $1.07(0.66-1.72)$ & 30 & $0.96(0.56-1.65)$ & 18 & $1.41(0.65-3.05)$ & 44 & $1.13(0.71-1.81)$ & 11 & $0.87(0.37-2.04)$ \\
\hline & 19 & $1.36(0.59-3.12)$ & 62 & $1.21(0.78-1.88)$ & 43 & $0.99(0.60-1.63)$ & 36 & $2.06(1.02-4.17)$ & 70 & $1.31(0.85-2.01)$ & 20 & $1.16(0.54-2.48)$ \\
\hline & 13 & $1.14(0.47-2.76)$ & 67 & $1.66(1.07-2.57)$ & 42 & $1.22(0.74-2.02)$ & 37 & $2.69(1.33-5.43)$ & 69 & $1.64(1.06-2.52)$ & 18 & $1.33(0.61-2.89)$ \\
\hline & & 0.547 & & 0.011 & & 0.390 & & $<0.001$ & & 0.013 & & 0.332 \\
\hline \multirow[t]{4}{*}{ Hip } & 9 & 1.00 & 32 & 1.00 & 32 & 1.00 & 8 & 1.00 & 36 & 1.00 & 11 & 1.00 \\
\hline & 13 & $1.36(0.58-3.18)$ & 32 & $0.95(0.58-1.55)$ & 26 & $0.77(0.46-1.29)$ & 18 & $2.12(0.92-4.88)$ & 44 & $1.15(0.74-1.79)$ & 7 & $0.62(0.241 .60)$ \\
\hline & 11 & $1.11(0.46-2.69)$ & 67 & $1.89(1.23-2.89)$ & 38 & $1.06(0.66-1.70)$ & 37 & $4.20(1.95-9.05)$ & 61 & $1.52(1.00-2.30)$ & 23 & $1.99(0.96-4.12)$ \\
\hline & 17 & $1.37(0.60-3.13)$ & 68 & $1.58(1.04-2.44)$ & 44 & $1.02(0.64-1.62)$ & 39 & $3.65(1.70-7.88)$ & 73 & $1.50(1.00-2.25)$ & 19 & $1.39(0.65-2.95)$ \\
\hline$p$-trend & & 0.577 & & 0.003 & & 0.619 & & $<0.001$ & & 0.025 & & 0.102 \\
\hline \multirow[t]{4}{*}{ Waist } & 12 & 1.00 & 34 & 1.00 & 32 & 1.00 & 11 & 1.00 & 41 & 1.00 & 10 & 1.00 \\
\hline & 11 & $0.80(0.35-1.83)$ & 27 & $0.71(0.43-1.17)$ & 20 & $0.55(0.31-0.96)$ & 16 & $1.30(0.61-2.81)$ & 33 & $0.71(0.45-1.12)$ & 9 & $0.81(0.33-1.99)$ \\
\hline & 12 & $0.80(0.36-1.79)$ & 63 & $1.53(1.00-2.32)$ & 44 & $1.10(0.70-1.74)$ & 31 & $2.34(0.65-2.30)$ & 67 & $1.32(0.90-1.96)$ & 17 & $1.47(0.67-3.23)$ \\
\hline & 15 & $1.00(0.46-2.16)$ & 75 & $1.90(1.26-2.87)$ & 44 & $1.13(0.72-1.80)$ & 44 & $3.51(1.80-6.83)$ & 73 & $1.50(1.02-2.22)$ & 24 & $2.18(0.04-4.60)$ \\
\hline$p$-trend & & 0.966 & & $<0.001$ & & 0.155 & & $<0.001$ & & 0.002 & & 0.009 \\
\hline \multirow[t]{4}{*}{ BMI } & 16 & 1.00 & 39 & 1.00 & 37 & 1.00 & 17 & 1.00 & 50 & 1.00 & 14 & 1.00 \\
\hline & 11 & $0.65(0.30-1.42)$ & 35 & $0.86(0.54-1.35)$ & 24 & $0.61(0.36-1.02)$ & 21 & $1.19(0.63-2.26)$ & 41 & $0.77(0.51-1.17)$ & 8 & $0.57(0.24-1.37)$ \\
\hline & 10 & $0.54(0.24-1.20)$ & 48 & $1.11(0.73-1.71)$ & 33 & $0.78(0.48-1.25)$ & 23 & $1.22(0.65-2.30)$ & 48 & $0.85(0.57-1.26)$ & 18 & $1.27(0.62-2.57)$ \\
\hline & & $0.78(0.37-1.64)$ & 77 & $2.04(1.38-3.03)$ & 46 & $1.22(0.79-1.91)$ & 41 & $2.51(1.41-4.46)$ & 75 & $1.51(1.05-2.17)$ & 20 & $1.62(0.80-3.24)$ \\
\hline$p$-trend & & 0.420 & & $<0.001$ & & 0.218 & & 0.001 & & 0.015 & & 0.058 \\
\hline \multirow[t]{4}{*}{ WHR } & 14 & 1.00 & 44 & 1.00 & 43 & 1.00 & 16 & 1.00 & 53 & 1.00 & 14 & 1.00 \\
\hline & 10 & $0.96(0.43-2.17)$ & 32 & $1.02(0.65-1.61)$ & 23 & $0.78(0.47-1.31)$ & 17 & $1.49(0.75-2.96)$ & 38 & $0.99(0.65-1.50)$ & 7 & $0.71(0.29-1.76)$ \\
\hline & 18 & $1.35(0.67-2.73)$ & 55 & $1.39(0.93-2.07)$ & 34 & $0.90(0.57-1.43)$ & 35 & $2.49(1.38-4.51)$ & 59 & $1.22(0.84-1.77)$ & 19 & $1.53(0.77-3.06)$ \\
\hline & 8 & $0.65(0.27-1.56)$ & 68 & $1.98(1.35-2.91)$ & 43 & $1.29(0.83-1.99)$ & 34 & $2.76(1.52-5.04)$ & 64 & $1.50(1.03-2.17)$ & 20 & 1.88(0.94-3.76) \\
\hline$p$-trend & & 0.631 & & $<0.001$ & & 0.251 & & $<0.001$ & & 0.021 & & 0.028 \\
\hline
\end{tabular}

Adjusted for age, level of education, smoking habits and alcohol consumption.

distribution, reflected as waist- and hip circumference, and colorectal cancer risk [6,8,12,13,18-22], but available epidemiologic evidence suggests that abdominal obesity (high waist circumference and waist-hip-ratio) may be more predictive of colorectal cancer risk than overall obesity $[6,8,12,13,18]$. Increased bodyweight has been suggested to be more closely related to abdominal obesity in men, and to gluteofemoral obesity in women [17].
The exact biologic mechanisms underlying the association between obesity and increased risk of CRC are not fully understood. Some authors have suggested components of the metabolic syndrome, in particular insulin-resistance and subsequent hyperinsulinemia, to be the underlying link, which may reflect the growthpromoting effects of insulin [32,34]. These speculations are also supported by studies that found an increased 
Table 5 Anthropometric factors and relative risk of colorectal cancer of different $T, N$ and $M$ stages in women

\begin{tabular}{|c|c|c|c|c|c|c|c|c|c|c|c|c|}
\hline $\begin{array}{l}\text { Anthropometric } \\
\text { factor (quartiles) }\end{array}$ & (n) & $\begin{array}{l}\text { RR of } \\
\text { Tstage } 1 \& 2 \\
n=63\end{array}$ & (n) & $\begin{array}{l}\text { RR of } \\
\text { Tstage } 3 \& 4 \\
n=205\end{array}$ & (n) & $\begin{array}{l}\text { RR of } \\
N \text {-stage } 0 \\
n=150\end{array}$ & (n) & $\begin{array}{l}\text { RR of } \\
N \text {-stage } 1 \& 2 \\
n=105\end{array}$ & (n) & $\begin{array}{l}\text { RR of } \\
M \text {-stage } 0 \\
n=236\end{array}$ & (n) & $\begin{array}{l}\text { RR of } \\
M \text {-stage } 1 \\
n=56\end{array}$ \\
\hline \multirow[t]{4}{*}{ Height } & 11 & 1.00 & 43 & 1.00 & 32 & 1.00 & 21 & 1.00 & 45 & 1.00 & 14 & 1.00 \\
\hline & 20 & $1.40(0.67-2.94)$ & 69 & 1.18(0.80-1.73) & 49 & $1.16(0.74-1.82)$ & 33 & $1.12(0.64-1.93)$ & 74 & $1.23(0.85-1.79)$ & 24 & $1.18(0.61-2.31)$ \\
\hline & 15 & $1.43(0.65-3.14)$ & 54 & $1.29(0.86-1.94)$ & 36 & $1.20(0.74-1.95)$ & 34 & 1.53(0.88-2.66) & 67 & $1.53(1.04-2.24)$ & 8 & $0.56(0.23-1.35)$ \\
\hline & 17 & $1.81(0.81-4.00)$ & 39 & $1.02(0.65-1.60)$ & 35 & $1.33(0.80-2.19)$ & 17 & $0.81(0.42-1.57)$ & 50 & $1.23(0.81-1.87)$ & 10 & $0.77(0.33-1.78)$ \\
\hline$p$-trend & & 0.168 & & 0.787 & & 0.274 & & 0.925 & & 0.184 & & 0.233 \\
\hline \multirow[t]{4}{*}{ Weight } & 8 & 1.00 & 31 & 1.00 & 23 & 1.00 & 18 & 1.00 & 32 & 1.00 & 12 & 1.00 \\
\hline & 16 & $1.71(0.73-4.00)$ & 54 & $1.49(0.96-2.32)$ & 39 & $1.45(0.88-2.46)$ & 30 & $1.40(0.78-2.52)$ & 62 & $1.66(1.09-2.55)$ & 19 & $1.34(0.65-2.76)$ \\
\hline & 22 & $2.67(1.18-6.07)$ & 54 & $1.74(1.18-2.72)$ & 43 & $1.90(1.14-3.16)$ & 27 & $1.51(0.83-2.74)$ & 71 & $2.21(1.45-3.37)$ & 7 & $0.59(0.23-1.49)$ \\
\hline & 17 & $1.85(0.79-4.32)$ & 66 & $1.70(1.10-2.62)$ & 47 & $1.69(1.02-2.80)$ & 30 & $1.35(0.75-2.43)$ & 71 & $1.85(2121-2.81)$ & 18 & $1.16(0.55-2.45)$ \\
\hline$p$-trend & & 0.114 & & 0.018 & & 0.034 & & 0.367 & & 0.004 & & 0.865 \\
\hline \multirow[t]{4}{*}{ Body fat percentage } & 11 & 1.00 & 28 & 1.00 & 26 & 1.00 & 14 & 1.00 & 37 & 1.00 & 10 & 1.00 \\
\hline & 15 & $1.25(0.57-2.77)$ & 37 & $1.26(0.77-2.07)$ & 31 & $1.13(0.67-1.91)$ & 18 & $1.29(0.64-2.60)$ & 44 & $1.13(0.72-1.76)$ & 11 & $1.07(0.45-2.52)$ \\
\hline & 15 & $1.11(0.51-2.45)$ & 57 & $1.71(1.08-2.69)$ & 40 & $1.25(0.76-2.05)$ & 31 & $1.98(1.05-3.74)$ & 66 & $1.50(1.00-2.25)$ & 13 & $1.09(0.47-2.51)$ \\
\hline & 22 & $1.15(0.55-2.42)$ & 83 & $1.71(1.11-2.66)$ & 55 & $1.17(0.73-1.90)$ & 42 & $1.92(1.03(3.57)$ & 89 & $1.42(0.96-2.11)$ & 22 & $1.26(0.58-2.72)$ \\
\hline$p$-trend & & 0.840 & & 0.009 & & 0.516 & & 0.021 & & 0.046 & & 0.539 \\
\hline \multirow[t]{4}{*}{ Hip } & 10 & 1.00 & 28 & 1.00 & 23 & 1.00 & 18 & 1.00 & 32 & 1.00 & 12 & 1.00 \\
\hline & 11 & $0.77(0.33-1.83)$ & 49 & $1.25(0.79-2.00)$ & 36 & $1.12(0.66-1.90)$ & 22 & $0.88(0.47-1.65)$ & 56 & $1.27(0.82-1.97)$ & 11 & $0.62(0.27-1.41)$ \\
\hline & 18 & $1.18(0.53-2.60)$ & 55 & 1.38(0.87-2.19) & 38 & $1.14(0.67-1.93)$ & 29 & $1.20(0.66-2.17)$ & 60 & $1.32(0.85-2.04)$ & 17 & $0.95(0.45-2.01)$ \\
\hline & 24 & 1.44(0.68-3.07) & 73 & $1.51(0.97-2.36)$ & 35 & $1.38(0.84-2.28)$ & 36 & $1.24(0.70-2.22)$ & 88 & $1.67(1.11-2.53)$ & 16 & $0.70(0.32-1.52)$ \\
\hline$p$-trend & & 0.149 & & 0.066 & & 0.184 & & 0.259 & & 0.012 & & 0.641 \\
\hline \multirow[t]{4}{*}{ Waist } & 9 & 1.00 & 32 & 1.00 & 24 & 1.00 & 18 & 1.00 & 32 & 1.00 & 12 & 1.00 \\
\hline & 13 & $0.95(0.40-2.26)$ & 43 & $0.97(0.61-1.55)$ & 34 & $0.99(0.58-1.68)$ & 22 & $0.93(0.50-1.74)$ & 54 & $1.20(0.77-1.87)$ & 11 & $0.67(0.30-1.53)$ \\
\hline & 22 & $1.53(0.70-3.34)$ & 67 & $1.36(0.89-2.08)$ & 47 & $1.23(0.75-2.01)$ & 37 & $1.41(0.80-2.50)$ & 79 & $1.61(1.06-2.43)$ & 16 & $0.86(0.40-1.83)$ \\
\hline & 19 & $1.33(0.59-2.97)$ & 63 & $1.23(0.80-1.90)$ & 47 & $1.17(0.71-1.94)$ & 28 & $1.08(0.59-2.00)$ & 71 & $1.43(0.94-2.19)$ & 17 & $0.86(0.40-1.85)$ \\
\hline$p$-trend & & 0.293 & & 0.161 & & 0.375 & & 0.491 & & 0.054 & & 0.933 \\
\hline \multirow[t]{4}{*}{ BMI } & 10 & 1.00 & 30 & 1.00 & 25 & 1.00 & 16 & 1.00 & 35 & 1.00 & 11 & 1.00 \\
\hline & 16 & $1.36(0.61-3.03)$ & 54 & $1.62(1.03-2.53)$ & 39 & $1.38(0.83-2.30)$ & 32 & $1.86(1.02-3.40)$ & 32 & $1.60(1.06-2.43)$ & 15 & $1.23(0.57-2.70)$ \\
\hline & 19 & $1.58(0.73-3.42)$ & 53 & $1.49(0.95-2.34)$ & 39 & $1.30(0.78-2.15)$ & 25 & $1.38(0.73-2.60)$ & 65 & $1.58(1.04-2.40)$ & 11 & $0.83(0.36-1.94)$ \\
\hline & 18 & $1.56(0.71-3.45)$ & 68 & $1.84(1.18-2.86)$ & 49 & $1.58(0.96-2.60)$ & 32 & $1.76(0.95-3.25)$ & 73 & $1.77(1.17-2.67)$ & 19 & $1.38(0.64-2.98)$ \\
\hline$p$-trend & & 0.259 & & 0.017 & & 0.107 & & 0.205 & & 0.016 & & 0.592 \\
\hline \multirow[t]{4}{*}{ WHR } & 17 & 1.00 & 50 & 1.00 & 40 & 1.00 & 25 & 1.00 & 66 & 1.00 & 14 & 1.00 \\
\hline & 15 & $1.11(0.55-2.24)$ & 41 & $0.98(0.65-1.48)$ & 35 & $1.04(0.66-1.64)$ & 22 & $1.04(0.59-1.85)$ & 54 & $1.07(0.74-1.54)$ & 9 & $0.78(0.34-1.80)$ \\
\hline & 15 & $0.90(0.44-1.82)$ & 57 & $1.11(0.76-1.64)$ & 33 & $0.78(0.49-1.24)$ & 34 & $1.38(0.82-2.32)$ & 55 & $0.90(0.62-1.30)$ & 20 & 1.38(0.69-2.76) \\
\hline & 16 & $1.01(0.53-2.12)$ & 57 & $1.24(0.84-1.82)$ & 44 & $1.15(0.74-1.78)$ & 24 & $1.10(0.63-1.94)$ & 66 & $1.18(0.83-1.68)$ & 13 & $1.07(0.50-2.30)$ \\
\hline$p$-trend & & 0.972 & & 0.220 & & 0.816 & & 0.488 & & 0.550 & & 0.525 \\
\hline
\end{tabular}

Adjusted for age, level of education, smoking habits and alcohol consumption.

risk of CRC in patients with Type 2 diabetes [35-37], as hyperinsulinemia is also related to increased levels of insulin-like growth factor 1 , which is known to have cancer promoting effects [38-40]. Further potential mediators include leptin, elevated serum levels of which have been demonstrated to stimulate growth of colonic epithelial cells [41-43], and adinopectin, that is secreted from visceral adipose tissue, correlates inversely to BMI, and has been demonstrated to have antiangionenic and antitumour activities [44,45]. Obesity, in particular abdominal obesity, is linked to insulin-resistance, hyperinsulinemia and Type 2 diabetes [46].

Several epidemiological studies have shown that elevated levels of estrogen and progesterone is associated with lower risk of developing CRC [47-49]. Recent studies found no reduced risk of CRC in women with higher 
Table 6 Anthropometric factors and relative risk of colon cancer in the full cohort, women and men

\begin{tabular}{|c|c|c|c|c|c|c|}
\hline $\begin{array}{l}\text { Anthropometric } \\
\text { factor (quartiles) }\end{array}$ & (n) & $\begin{array}{l}R R \text { all } \\
n=337\end{array}$ & (n) & $\begin{array}{l}\text { RR men } \\
n=157\end{array}$ & (n) & $\begin{array}{l}\text { RR women } \\
n=180\end{array}$ \\
\hline \multirow[t]{4}{*}{ Height } & 76 & 1.00 & 32 & 1.00 & 37 & 1.00 \\
\hline & 77 & $1.02(0.74-1.40)$ & 37 & $1.27(0.79-2.04)$ & 59 & $1.23(0.81-1.86)$ \\
\hline & 82 & $1.10(0.80-1.52)$ & 40 & $1.07(0.67-1.72)$ & 44 & $1.31(0.84-2.04)$ \\
\hline & 102 & $1.37(1.00-1.88)$ & 48 & $1.52(0.96-2.42)$ & 40 & 1.33(0.83-2.12) \\
\hline p-trend & & 0.041 & & 0.137 & & 0.221 \\
\hline \multirow[t]{4}{*}{ Weight } & 65 & 1.00 & 27 & 1.00 & 31 & 1.00 \\
\hline & 72 & $1.11(0.79-1.56)$ & 38 & $1.33(0.81-2.19)$ & 43 & $1.18(0.74-1.88)$ \\
\hline & 91 & $1.23(0.89-1.70)$ & 39 & $1.40(0.86-2.30)$ & 51 & $1.59(1.01-2.00)$ \\
\hline & 109 & $1.51(1.10-2.07)$ & 53 & 1.96(1.22-3.14) & 55 & $1.39(0.89-2.17)$ \\
\hline$p$-trend & & 0.007 & & 0.005 & & 0.088 \\
\hline \multirow[t]{4}{*}{ Body fat percentage } & 72 & 1.00 & 24 & 1.00 & 30 & 1.00 \\
\hline & 84 & $1.09(0.79-1.49)$ & 34 & $1.10(0.65-1.86)$ & 38 & $1.14(0.70-1.85)$ \\
\hline & 77 & $1.00(0.72-1.39)$ & 44 & $1.05(0.64-1.73)$ & 44 & $1.15(0.72-1.83)$ \\
\hline & 107 & $1.04(0.76-1.43)$ & 54 & $1.64(1.01-2.67)$ & 68 & $1.19(0.77-1.85)$ \\
\hline p-trend & & 0.880 & & 0.039 & & 0.470 \\
\hline \multirow[t]{4}{*}{ Hip } & 59 & 1.00 & 29 & 1.00 & 30 & 1.00 \\
\hline & 66 & $0.92(0.65-1.31)$ & 23 & $0.74(0.43-1.28)$ & 39 & $0.89(0.55-1.43)$ \\
\hline & 99 & $1.31(0.94-1.82)$ & 49 & $1.51(0.95-2.40)$ & 54 & $1.15(0.73-1.82)$ \\
\hline & 113 & $1.23(0.89-1.70)$ & 56 & $1.42(0.90-2.24)$ & 68 & $1.00(0.63-1.57)$ \\
\hline$p$-trend & & 0.049 & & 0.019 & & 0.725 \\
\hline \multirow[t]{4}{*}{ Waist } & 60 & 1.00 & 27 & 1.00 & 31 & 1.00 \\
\hline & 76 & $1.12(0.79-1.58)$ & 22 & $0.72(0.41-1.27)$ & 38 & $0.83(0.51-1.35)$ \\
\hline & 71 & $0.92(0.65-1.31)$ & 51 & $1.57(0.98-2.50)$ & 58 & $1.15(0.74-1.79)$ \\
\hline & 130 & $1.63(1.18-2.24)$ & 57 & $1.84(1.16-2.92)$ & 53 & $0.98(0.62-1.55)$ \\
\hline$p$-trend & & 0.003 & & $<0.001$ & & 0.671 \\
\hline \multirow[t]{4}{*}{ Bmi } & 56 & 1.00 & 33 & 1.00 & 30 & 1.00 \\
\hline & 65 & $1.13(0.79-1.63)$ & 29 & $0.85(0.51-1.40)$ & 40 & $1.13(0.70-1.82)$ \\
\hline & 106 & $1.39(1.00-1.93)$ & 40 & $1.10(0.69-1.76)$ & 57 & $1.52(0.97-2.37)$ \\
\hline & 110 & $1.61(1.16-2.24)$ & 55 & $1.78(1.14-2.74)$ & 53 & $1.33(084-2.10)$ \\
\hline$p$-trend & & 0.001 & & 0.004 & & 0.134 \\
\hline \multirow[t]{4}{*}{ Whr } & 73 & 1.00 & 32 & 1.00 & 48 & 1.00 \\
\hline & 77 & $1.05(0.76-1.45)$ & 24 & $1.06(0.63-1.81)$ & 33 & $0.85(0.54-1.32)$ \\
\hline & 75 & $0.96(0.69-1.33)$ & 52 & $1.84(1.18-2.87)$ & 51 & $1.10(0.71-1.58)$ \\
\hline & 112 & $1.61(1.18-2.20)$ & 49 & $2.01(1.28-3.16)$ & 48 & $1.9(0.72-1.64)$ \\
\hline p-trend & & 0.005 & & $<0.001$ & & 0.509 \\
\hline
\end{tabular}

Adjusted for age, level of education, smoking habits and alcohol consumption.

levels of estradiol and estrone, suggesting that progesterone is the key factor for reduction of CRC risk in women [50-52]. Use of hormone replacement therapy (HRT) in post-menopausal women could in part explain sexrelated differences in the association between adiposity and CRC risk, as BMI is positively associated with circulating levels of estradiol in postmenopausal women and HRT has been associated with a reduced risk of CRC in observational and interventional studies [53-56]. The associations between sex hormones and risk of CRC in men is poorly understood, but two studies have supported the hypothesis that lower androgenecity may increase men's risk of developing CRC [57,58]. Lower androgen levels seem to be more frequent in obese men, and treatment with testosterone reduces insulin resistance, suggesting a role of androgens in promoting insuline sensitivity and hereby one possible mechanism in the development of CRC. 
Table 7 Anthropometric factors and relative risk of rectal cancer in the full cohort, women and men

\begin{tabular}{|c|c|c|c|c|c|c|}
\hline $\begin{array}{l}\text { Anthropometric } \\
\text { factor (quartiles) }\end{array}$ & (n) & $\begin{array}{l}R R \text { all } \\
n=213\end{array}$ & (n) & $\begin{array}{l}\text { RR men } \\
n=107\end{array}$ & (n) & $\begin{array}{l}\text { RR women } \\
n=106\end{array}$ \\
\hline \multirow[t]{4}{*}{ Height } & 46 & 1.00 & 26 & 1.00 & 19 & 1.00 \\
\hline & 46 & $0.93(0.61-1.40)$ & 27 & $1.13(0.66-1.93)$ & 37 & $1.36(0.78-2.37)$ \\
\hline & 61 & $1.22(0.83-1.81)$ & 28 & $0.93(0.55-1.60)$ & 27 & $1.31(0.72-2.37)$ \\
\hline & 61 & $1.16(0.78-1.74)$ & 26 & $1.03(0.59-1.80)$ & 23 & $1.18(0.63-2.21)$ \\
\hline p-trend & & 0.273 & & 0.902 & & 0.713 \\
\hline \multirow[t]{4}{*}{ Weight } & 37 & 1.00 & 24 & 1.00 & 12 & 1.00 \\
\hline & 52 & $1.45(0.95-2.21)$ & 26 & $1.04(0.60-1.82)$ & 33 & $2.36(1.22-4.58)$ \\
\hline & 54 & $1.27(0.83-1.94)$ & 26 & $1.06(0.60-1.85)$ & 27 & $2.34(1.18-4.62)$ \\
\hline & 71 & $1.68(1.12-2.53)$ & 31 & $1.26(0.73-2.16)$ & 34 & $2.46(1.27-4.77)$ \\
\hline$p$-trend & & 0.029 & & 0.411 & & 0.022 \\
\hline \multirow[t]{4}{*}{ Body fatper centage } & 51 & 1.00 & 15 & 1.00 & 15 & 1.00 \\
\hline & 55 & $1.03(0.70-1.50)$ & 19 & $0.98(0.50-1.94)$ & 18 & $1.26(0.63-2.51)$ \\
\hline & 38 & $0.73(0.48-1.12)$ & 44 & $1.64(0.91-2.95)$ & 33 & $2.05(1.10-3.80)$ \\
\hline & 70 & $1.05(0.72-1.53)$ & 28 & $1.31(0.70-2.47)$ & 40 & $1.83(1.00-3.37)$ \\
\hline$p$-trend & & 0.956 & & 0.173 & & 0.026 \\
\hline \multirow[t]{4}{*}{ Hip } & 33 & 1.00 & 17 & 1.00 & 13 & 1.00 \\
\hline & 43 & $1.13(0.72-1.78)$ & 26 & $1.48(0.80-2.73)$ & 26 & $1.52(0.78-2.97)$ \\
\hline & 61 & $1.59(103-2.44)$ & 31 & $1.71(0.94-3.10)$ & 24 & $1.45(0.74-2.88)$ \\
\hline & 77 & $1.69(1.12-2.57)$ & 33 & $1.52(0.84-2.76)$ & 43 & $2.30(1.22-4.34)$ \\
\hline$p$-trend & & 0.003 & & 0.180 & & 0.009 \\
\hline \multirow[t]{4}{*}{ Waist } & 31 & 1.00 & 23 & 1.00 & 10 & 1.00 \\
\hline & 53 & $1.50(0.96-2.34)$ & 18 & $0.70(0.38-1.30)$ & 29 & $2.24(1.09-4.60)$ \\
\hline & 48 & $1.21(0.76-1.91)$ & 32 & $1.14(0.67-1.96)$ & 34 & $2.38(1.17-4.83)$ \\
\hline & 82 & $1.96(1.27-3.00)$ & 34 & $1.26(0.74-2.169$ & 33 & $2.43(1.19-4.98)$ \\
\hline$p$-trend & & 0.005 & & 0.160 & & 0.032 \\
\hline \multirow[t]{4}{*}{ Bmi } & 39 & 1.00 & 30 & 1.00 & 15 & 1.00 \\
\hline & 50 & $1.31(0.86-1.99)$ & 18 & $0.57(0.32-1.03)$ & 35 & $2.22(1.21-4.08)$ \\
\hline & 51 & $0.99(0.65-1.51)$ & 24 & $0.72(0.42-1.23)$ & 22 & $1.34(0.69-2.61)$ \\
\hline & 74 & $1.62(1.09-2.42)$ & 35 & $1.16(0.70-1.91)$ & 34 & $2.17(1.17-4.04)$ \\
\hline$p$-trend & & 0.044 & & 0.407 & & 0.093 \\
\hline \multirow[t]{4}{*}{ Whr } & 44 & 1.00 & 33 & 1.00 & 29 & 1.00 \\
\hline & 43 & $0.96(0.63-1.47)$ & 21 & $0.86(0.50-1.49)$ & 28 & $1.36(0.79-2.34)$ \\
\hline & 64 & $1.31(0.89-1.94)$ & 24 & $0.77(0.45-1.31)$ & 25 & $1.02(0.58-1.79)$ \\
\hline & 62 & $1.32(0.88-1.99)$ & 29 & 1.04(0.62-1.72) & 29 & $1.35(0.79-2.34)$ \\
\hline p-trend & & 0.082 & & 0.952 & & 0.475 \\
\hline
\end{tabular}

Adjusted for age, level of education, smoking habits and alcohol consumption.

In agreement with most previous reports, we found a stronger association between body size and risk of colon compared to rectal cancer in general, in particular in men [4]. In line with these findings, a meta-analysis showed that physical activity, which is related to improved insulin sensitivity $[59,60]$, was associated with a reduced risk of colon cancer, but not of rectal cancer [61]. This may suggest that insulin resistance, hyperinsulinemia and other factors related to obesity are stronger risk factors for colon than rectal cancer. Several prospective studies reported that circulating C-peptide $[39,42,62,63]$ and leptin $[42,43]$ concentrations were more strongly associated with risk of colon cancer than overall colorectal cancer or rectal cancer. Our findings of a significant association between high weight, bodyfat percentage, hip and waist, and an increased risk of rectal cancer in women but not men are in contrast to most previous studies, including a large meta-analysis 
demonstrating a statistically significant association between obesity and an increased risk of rectal cancer in men [4]. While these findings indeed suggest differences in tumour susceptibility related to location, the potential mechanisms accounting for these differences need further investigation.

A limitation to the present study is the relatively small number of cases in some subgroups, i.e. it is possible that some of the non-significant findings in relation to gender or tumour stages may have been caused by a potential type II error. Therefore, these data need to be validated in larger patient cohorts. It should also be pointed out that since mean age at diagnosis was $>70$ years for women [27], interpretations relying on sex as evidence for influence of sex hormones must be made with caution until further knowledge has been gained. To this end, future studies are warranted to explore the effects of e.g. hormone replacement therapy on clinicopathological factors, molecular correlates and survival from CRC.

Certain methodological aspects need further attention. The validity of the anthropometric measurements is one aspect as there may be a potential inter-observer variation. Recommendations for the nurses performing baseline examinations described how participants should be dressed, in which position the participants should be examined, and location for the estimation of waist- and hip measurements. We therefore consider the risk of misclassification of anthropometric measurements to be low. In contrast, most previous studies have used selfreported anthropometric measures.

It is also possible that participation in the MDCS was associated with body constitution, which may have lead to potential selection bias. In a previous paper, Manjer et al [25] compared BMI in the MDCS in relation to the background population, and found an equal distribution of overweight/obesity.

Another aspect is the validity of collected data. As anthropometric data was assessed only at baseline, it is possible that some individuals have gained and some have lost weight. Such a misclassification is likely to lead to an attenuation of risks and, if anything, observed risks may be underestimated.

In addition, it might be difficult to apply incidence rates from the MDCS to the background population as participants may to some degree have been selected in terms of socio-economic factors and risk of CRC. Nevertheless, we consider it possible to make internal comparisons comparing subjects with high versus low levels of the study measurements in order to obtain relative risks.

\section{Conclusion}

This study is the first to show a relationship between obesity, measured as several different anthropometric factors, and an increased risk of colorectal cancer of more advanced clinical stage, in particular in men. These findings need to be confirmed in future studies, as they may be of importance in the development of novel strategies for prevention of the disease, such as tailored screening programmes taking sex and anthropometric factors into account.

\section{Additional file}

Additional file 1: Distribution of quartiles of anthropometric measurements.

\section{Abbreviations}

CRC: Colorectal cancer; MDCS: Malmö diet and cancer study; BMI: Body mass index; WHR: Waist-hip ratio; T-stage: Tumour stage; N-stage: Nodal stage;

M-stage: Metastatic stage; HRT: Hormone replacement therapy.

\section{Competing interests}

The authors declare that no competing interests exist.

\section{Authors' contributions}

JB performed the statistical analyses and drafted the manuscript. SW, BN and AG collected clinical data. JM assisted with the statistical analyses and helped draft the manuscript KJ conceived of the study, carried out the

histopathological re-evaluation, and helped draft the manuscript. All authors read and approved the final manuscript.

\section{Acknowledgments}

This study was supported by grants from the Knut and Alice Wallenberg Foundation, the Swedish Cancer Society, the Gunnar Nilsson Cancer Foundation and the Research Funds of Skåne University Hospital.

\section{Author details}

Department of Clinical Sciences, Division of Pathology, Lund University, Skåne University Hospital, Lund SE-221 85, Sweden. ${ }^{2}$ Department of Clinical Sciences, Division of Surgery, Lund University, Skåne University Hospital, Malmö SE-205 02, Sweden. ${ }^{3}$ The Malmö Diet and Cancer Study, Lund University, Malmö SE-205 02, Sweden.

Received: 16 July 2012 Accepted: 22 September 2012

Published: 16 October 2012

\section{References}

1. Parkin DM, Bray F, Ferlay J, Pisani P: Global cancer statistics, 2002. CA Cancer J Clin 2005, 55(2):74-108.

2. Eheman C, Henley SJ, Ballard-Barbash R, Jacobs EJ, Schymura MJ, Noone AM, Pan L, Anderson RN, Fulton JE, Kohler BA, et al: Annual Report to the Nation on the status of cancer, 1975-2008, featuring cancers associated with excess weight and lack of sufficient physical activity. Cancer 2012, 118(9):2338-2366.

3. Dai Z, Xu YC, Niu L: Obesity and colorectal cancer risk: a meta-analysis of cohort studies. World J Gastroenterol 2007, 13(31):4199-4206.

4. Larsson SC, Wolk A: Obesity and colon and rectal cancer risk: a metaanalysis of prospective studies. Am J Clin Nutr 2007, 86(3):556-565.

5. Renehan AG, Tyson M, Egger M, Heller RF, Zwahlen M: Body-mass index and incidence of cancer: a systematic review and meta-analysis of prospective observational studies. Lancet 2008, 371(9612):569-578.

6. Pischon T, Lahmann PH, Boeing H, Friedenreich C, Norat T, Tjonneland A, Halkjaer J, Overvad K, Clavel-Chapelon F, Boutron-Ruault MC, et al: Body size and risk of colon and rectal cancer in the European Prospective Investigation Into Cancer and Nutrition (EPIC). J Natl Cancer Inst 2006, 98(13):920-931.

7. Terry PD, Miller AB, Rohan TE: Obesity and colorectal cancer risk in women. Gut 2002, 51(2):191-194. 
8. Giovannucci E, Ascherio A, Rimm EB, Colditz GA, Stampfer MJ, Willett WC: Physical activity, obesity, and risk for colon cancer and adenoma in men. Ann Intern Med 1995, 122(5):327-334.

9. Murphy TK, Calle EE, Rodriguez C, Kahn HS, Thun MJ: Body mass index and colon cancer mortality in a large prospective study. Am J Epidemiol 2000 152(9):847-854.

10. Lin J, Zhang SM, Cook NR, Rexrode KM, Lee IM, Buring JE: Body mass index and risk of colorectal cancer in women (United States). Cancer Causes Control 2004, 15(6):581-589.

11. Terry P, Giovannucci E, Bergkvist L, Holmberg L, Wolk A: Body weight and colorectal cancer risk in a cohort of Swedish women: relation varies by age and cancer site. Br J Cancer 2001, 85(3):346-349.

12. Moore LL, Bradlee ML, Singer MR, Splansky GL, Proctor MH, Ellison RC, Kreger BE: BMI and waist circumference as predictors of lifetime colon cancer risk in Framingham Study adults. Int J Obes Relat Metab Disord 2004, 28(4):559-567.

13. Maclnnis RJ, English DR, Hopper JL, Haydon AM, Gertig DM, Giles GG: Body size and composition and colon cancer risk in men. Cancer Epidemiol Biomarkers Prev 2004, 13(4):553-559.

14. Kune GA, Kune S, Watson LF: Body weight and physical activity as predictors of colorectal cancer risk. Nutr Cancer 1990, 13(1-2):9-17.

15. Caan BJ, Coates AO, Slattery ML, Potter JD, Quesenberry SM Jr, Edwards CP. Body size and the risk of colon cancer in a large case-control study. Int J Obes Relat Metab Disord 1998, 22(2):178-184.

16. Russo A, Franceschi S, La Vecchia C, Dal Maso L, Montella M, Conti E, Giacosa A, Falcini F, Negri E: Body size and colorectal-cancer risk. Int J Cancer 1998, 78(2):161-165.

17. Krotkiewski M, Bjorntorp P, Sjostrom L, Smith U: Impact of obesity on metabolism in men and women. Importance of regional adipose tissue distribution. J Clin Invest 1983, 72(3):1150-1162.

18. Maclnnis RJ, English DR, Hopper JL, Gertig DM, Haydon AM, Giles GG: Body size and composition and colon cancer risk in women. Int J Cancer 2006, 118(6):1496-1500.

19. Schoen RE, Tangen CM, Kuller LH, Burke GL, Cushman M, Tracy RP, Dobs A Savage PJ: Increased blood glucose and insulin, body size, and incident colorectal cancer. J Natl Cancer Inst 1999, 91(13):1147-1154.

20. Martinez ME, Giovannucci E, Spiegelman D, Hunter DJ, Willett WC, Colditz GA: Leisure-time physical activity, body size, and colon cancer in women. Nurses' Health Study Research Group. J Natl Cancer Inst 1997 89(13):948-955.

21. Folsom AR, Kushi LH, Anderson KE, Mink PJ, Olson JE, Hong CP, Sellers TA, Lazovich D, Prineas RJ: Associations of general and abdominal obesity with multiple health outcomes in older women: the lowa Women's Health Study. Arch Intern Med 2000, 160(14):2117-2128

22. Larsson SC, Rutegard J, Bergkvist L, Wolk A: Physical activity, obesity, and risk of colon and rectal cancer in a cohort of Swedish men. Eur J Cancer 2006, 42(15):2590-2597.

23. Bostick RM, Potter JD, Kushi LH, Sellers TA, Steinmetz KA, McKenzie DR Gapstur SM, Folsom AR: Sugar, meat, and fat intake, and non-dietary risk factors for colon cancer incidence in lowa women (United States). Cancer Causes Control 1994, 5(1):38-52.

24. Riboli E, Kaaks R: The EPIC Project: rationale and study design. European Prospective Investigation into Cancer and Nutrition. Int J Epidemiol 1997, 26(Suppl 1):S6-S14.

25. Manjer J, Carlsson S, Elmstahl S, Gullberg B, Janzon L, Lindstrom M, Mattisson I, Berglund G: The Malmo Diet and Cancer Study: representativity, cancer incidence and mortality in participants and non-participants. Eur J Cancer Prev 2001, 10(6):489-499.

26. Steen B, Bosaeus I, Elmstahl S, Galvard H, Isaksson B, Robertsson E: Body composition in the elderly estimated with an electrical impedance method. Compr Gerontol A 1987, 1(3):102-105

27. Wangefjord S, Manjer J, Gaber A, Nodin B, Eberhard J, Jirstrom K: Cyclin D1 expression in colorectal cancer is a favorable prognostic factor in men but not in women in a prospective, population-based cohort study. Biol Sex Differ 2011, 2:10.

28. Larsson A, Johansson ME, Wangefjord S, Gaber A, Nodin B, Kucharzewska P, Welinder C, Belting M, Eberhard J, Johnsson A, et al: Overexpression of podocalyxin-like protein is an independent factor of poor prognosis in colorectal cancer. Br J Cancer 2011, 105(5):666-672.

29. Eberhard J, Gaber A, Wangefjord S, Nodin B, Uhlén M, Ericson Lindquist K, Jirström K: A cohort study of the prognostic and treatment predictive value of SATB2 expression in colorectal cancer. Br J Cancer 2012, 106(5):931-938.

30. Nodin B, Johannesson H, Wangefjord S, DP OC, Ericson-Lindquist $K$, Uhlen $M$, Jirstrom K, Eberhard J: Molecular correlates and prognostic significance of SATB1 expression in colorectal cancer. Diagn Pathol 2012, 7(1):115

31. Katz MH, Hauck WW: Proportional hazards (Cox) regression. J Gen Intern Med 1993, 8(12):702-711

32. Giovannucci E: Diet, body weight, and colorectal cancer: a summary of the epidemiologic evidence. J Womens Health (Larchmt) 2003, 12(2):173-182.

33. Karastergiou K, Fried SK: Sex differences in human adipose tissues - the biology of pear shape. Biol Sex Differ 2012, 3(1):13.

34. Giovannucci E: Insulin, insulin-like growth factors and colon cancer: a review of the evidence. J Nutr 2001, 131(11 Suppl):3109S-3120S.

35. Hu FB, Manson JE, Liu S, Hunter D, Colditz GA, Michels KB, Speizer FE, Giovannucci E: Prospective study of adult onset diabetes mellitus (type 2) and risk of colorectal cancer in women. J Natl Cancer Inst 1999, 91(6):542-547

36. McKeown-Eyssen G: Epidemiology of colorectal cancer revisited: are serum triglycerides and/or plasma glucose associated with risk? Cancer Epidemiol Biomarkers Prev 1994, 3(8):687-695.

37. Seow A, Yuan JM, Koh WP, Lee HP, Yu MC: Diabetes mellitus and risk of colorectal cancer in the Singapore Chinese Health Study. J Natl Cancer Inst 2006, 98(2):135-138.

38. Aaronson SA: Growth factors and cancer. Science 1991, 254(5035):1146-1153.

39. Kaaks R, Toniolo P, Akhmedkhanov A, Lukanova A, Biessy C, Dechaud H, Rinaldi S, Zeleniuch-Jacquotte A, Shore RE, Riboli E: Serum C-peptide, insulin-like growth factor (IGF)-I, IGF-binding proteins, and colorectal cancer risk in women. J Natl Cancer Inst 2000, 92(19):1592-1600.

40. Sandhu MS, Dunger DB, Giovannucci EL: Insulin, insulin-like growth factor-I (IGF-I), IGF binding proteins, their biologic interactions, and colorectal cancer. J Nat/ Cancer Inst 2002, 94(13):972-980

41. Hardwick JC, Van Den Brink GR, Offerhaus GJ, Van Deventer SJ, Peppelenbosch MP: Leptin is a growth factor for colonic epithelial cells. Gastroenterology 2001, 121(1):79-90.

42. Stattin P, Lukanova A, Biessy C, Soderberg S, Palmqvist R, Kaaks R, Olsson T, Jellum E: Obesity and colon cancer: does leptin provide a link? Int J Cancer 2004, 109(1):149-152.

43. Stattin P, Palmqvist R, Soderberg S, Biessy C, Ardnor B, Hallmans G, Kaaks R, Olsson T: Plasma leptin and colorectal cancer risk: a prospective study in Northern Sweden. Oncol Rep 2003, 10(6):2015-2021.

44. Brakenhielm E, Veitonmaki N, Cao R, Kihara S, Matsuzawa Y, Zhivotovsky B, Funahashi T, Cao Y: Adiponectin-induced antiangiogenesis and antitumor activity involve caspase-mediated endothelial cell apoptosis. Proc Natl Acad Sci USA 2004, 101(8):2476-2481.

45. Wei EK, Giovannucci E, Fuchs CS, Willett WC, Mantzoros CS: Low plasma adiponectin levels and risk of colorectal cancer in men: a prospective study. J Natl Cancer Inst 2005, 97(22):1688-1694.

46. Kahn BB, Flier JS: Obesity and insulin resistance. J Clin Invest 2000, 106(4):473-481.

47. La Vecchia C, Franceschi S: Reproductive factors and colorectal cancer. Cancer Causes Control 1991, 2(3):193-200.

48. Fernandez E, La Vecchia C, Balducci A, Chatenoud L, Franceschi S, Negri E: Oral contraceptives and colorectal cancer risk: a meta-analysis. $\mathrm{Br} \mathrm{J}$ Cancer 2001, 84(5):722-727.

49. Grodstein F, Newcomb PA, Stampfer MJ: Postmenopausal hormone therapy and the risk of colorectal cancer: a review and meta-analysis. Am J Med 1999, 106(5):574-582.

50. Gunter MJ, Hoover DR, Yu H, Wassertheil-Smoller S, Rohan TE, Manson JE, Howard BV, Wylie-Rosett J, Anderson GL, Ho GY, et al: Insulin, insulin-like growth factor-I, endogenous estradiol, and risk of colorectal cancer in postmenopausal women. Cancer Res 2008 68(1):329-337

51. Clendenen TV, Koenig KL, Shore RE, Levitz M, Arslan AA, ZeleniuchJacquotte A: Postmenopausal levels of endogenous sex hormones and risk of colorectal cancer. Cancer Epidemiol Biomarkers Prev 2009, 18(1):275-281.

52. Ritenbaugh C, Stanford JL, Wu L, Shikany JM, Schoen RE, Stefanick ML, Taylor V, Garland C, Frank G, Lane D, et al: Conjugated equine estrogens and colorectal cancer incidence and survival: the Women's Health 
Initiative randomized clinical trial. Cancer Epidemiol Biomarkers Prev 2008, 17(10):2609-2618.

53. Nelson HD, Humphrey LL, Nygren P, Teutsch SM, Allan JD: Postmenopausal hormone replacement therapy: scientific review. JAMA 2002, 288(7):872-881.

54. Rossouw JE, Anderson GL, Prentice RL, LaCroix AZ, Kooperberg C, Stefanick ML, Jackson RD, Beresford SA, Howard BV, Johnson KC, et al: Risks and benefits of estrogen plus progestin in healthy postmenopausal women: principal results From the Women's Health Initiative randomized controlled trial. JAMA 2002, 288(3):321-333.

55. Anderson GL, Limacher M, Assaf AR, Bassford T, Beresford SA, Black H, Bonds $D$, Brunner R, Brzyski R, Caan B, et al: Effects of conjugated equine estrogen in postmenopausal women with hysterectomy: the Women's Health Initiative randomized controlled trial. JAMA 2004 291(14):1701-1712.

56. Chlebowski RT, Wactawski-Wende J, Ritenbaugh C, Hubbell FA, Ascensao J, Rodabough RJ, Rosenberg CA, Taylor VM, Harris R, Chen C, et al: Estrogen plus progestin and colorectal cancer in postmenopausal women. $N$ Engl J Med 2004, 350(10):991-1004.

57. Slattery ML, Sweeney C, Murtaugh M, Ma KN, Wolff RK, Potter JD, Caan BJ, Samowitz W: Associations between ERalpha, ERbeta, and AR genotypes and colon and rectal cancer. Cancer Epidemiol Biomarkers Prev 2005, 14(12):2936-2942.

58. Alberg AJ, Gordon GB, Hoffman SC, Comstock GW, Helzlsouer KJ: Serum dehydroepiandrosterone and dehydroepiandrosterone sulfate and the subsequent risk of developing colon cancer. Cancer Epidemiol Biomarkers Prev 2000, 9(5):517-521.

59. Houmard JA, Tanner CJ, Slentz CA, Duscha BD, McCartney JS, Kraus WE: Effect of the volume and intensity of exercise training on insulin sensitivity. J Appl Physiol 2004, 96(1):101-106.

60. IVy JL: Role of exercise training in the prevention and treatment of insulin resistance and non-insulin-dependent diabetes mellitus. Sports Med 1997, 24(5):321-336.

61. Samad AK, Taylor RS, Marshall T, Chapman MA: A meta-analysis of the association of physical activity with reduced risk of colorectal cancer. Colorectal Dis 2005, 7(3):204-213.

62. Shimizu N, Nagata C, Shimizu H, Kametani M, Takeyama N, Ohnuma T, Matsushita S: Height, weight, and alcohol consumption in relation to the risk of colorectal cancer in Japan: a prospective study. Br J Cancer 2003, 88(7):1038-1043

63. Jenab M, Riboli E, Cleveland RJ, Norat T, Rinaldi S, Nieters A, Biessy C, Tjonneland A, Olsen A, Overvad K, et al: Serum C-peptide, IGFBP-1 and IGFBP-2 and risk of colon and rectal cancers in the European Prospective Investigation into Cancer and Nutrition. Int J Cancer 2007, 121(2):368-376.

doi:10.1186/2042-6410-3-23

Cite this article as: Brändstedt et al:: Gender, anthropometric factors and risk of colorectal cancer with particular reference to tumour location and TNM stage: a cohort study. Biology of Sex Differences 2012 3:23.

\section{Submit your next manuscript to BioMed Central and take full advantage of:}

- Convenient online submission

- Thorough peer review

- No space constraints or color figure charges

- Immediate publication on acceptance

- Inclusion in PubMed, CAS, Scopus and Google Scholar

- Research which is freely available for redistribution

Submit your manuscript at www.biomedcentral.com/submit
Ciomed Central 